\title{
A functional assay-based strategy for nanomaterial risk forecasting
}

$5 \quad{ }^{1}$ Christine Ogilvie Hendren

6 Center for the Environmental Implications of NanoTechnology

7 Duke University

8 Durham, NC 27708

9 Phone: 919.660 .5193

Email: christine.hendren@duke.edu

${ }^{2}$ Gregory V. Lowry

Department of Civil and Environmental Engineering

Carnegie Mellon University

119 Porter Hall

Pittsburgh, PA 15213

Phone: 412.268.2948

Email: glowry@andrew.cmu.edu

${ }^{3}$ Jason M. Unrine

Department of Plant and Soil Sciences

University of Kentucky

Agricultural Science Center

Lexington, KY 40546

Phone: 859.257.1657

Email: jason.unrine@uky.edu

${ }^{4}$ Mark R. Wiesner

Department of Civil and Environmental Engineering

Duke University

121 Hudson Hall PO Box 90287

Durham, NC 27708

Phone: 919.660 .5192

Email:wiesner@duke.edu

**Corresponding author 


\section{Graphical Abstract}

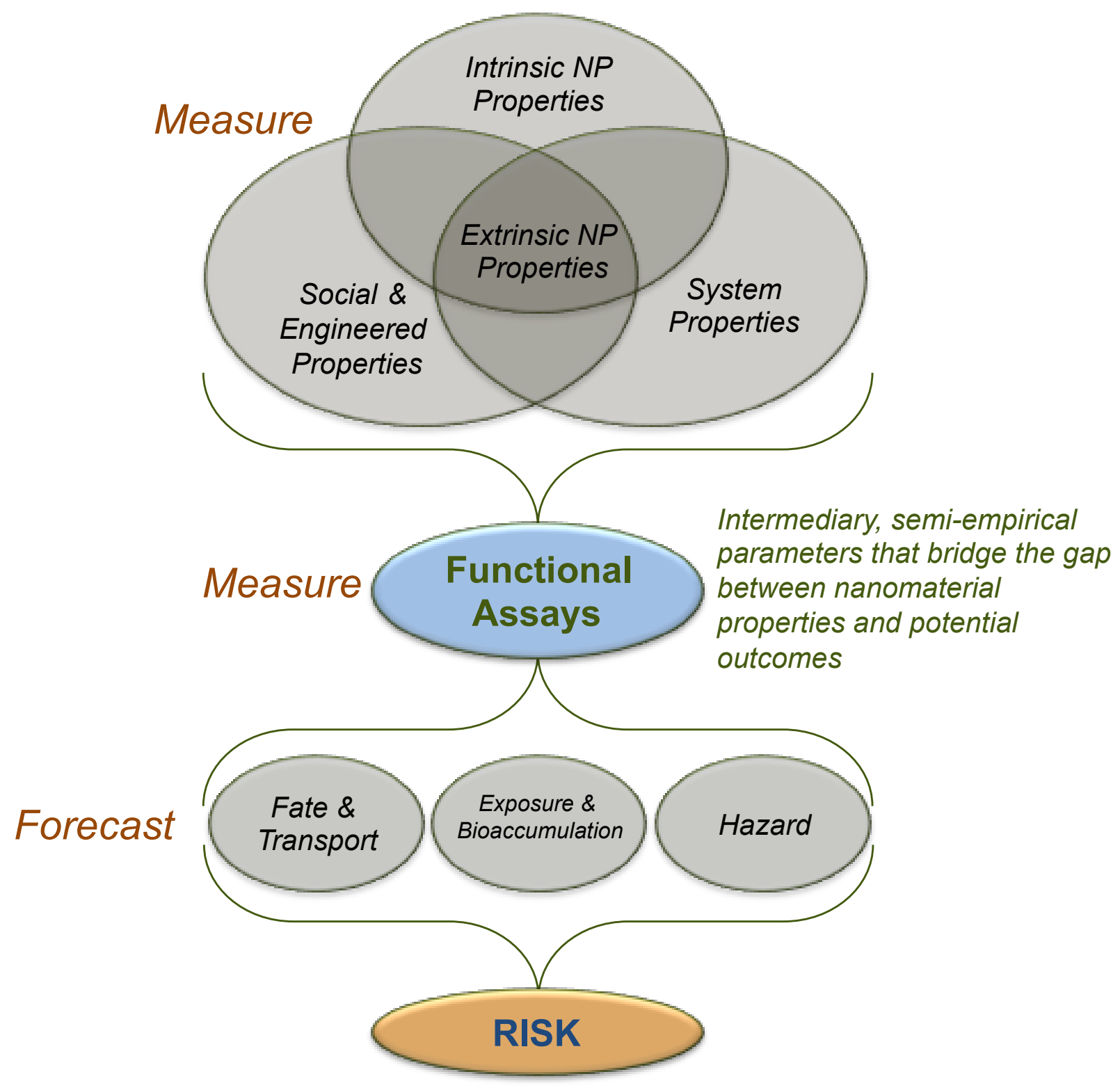




\section{Abstract}

41 The study of nanomaterial impacts on environment, health and safety (nanoEHS) has been

42 largely predicated on the assumption that exposure and hazard can be predicted from physical-

43 chemical properties of nanomaterials. This approach is rooted in the view that nanoöbjects

44 essentially resemble chemicals with additional particle-based attributes that must be included

45 among their intrinsic physical-chemical descriptors. With the exception of the trivial case of

46 nanomaterials made from toxic or highly reactive materials, this approach has yielded few

47 actionable guidelines for predicting nanomaterial risk. This article addresses inherent problems

48 in structuring a nanoEHS research strategy based on the goal of predicting outcomes directly

49 from nanomaterial properties, and proposes a framework for organizing data and designing

50 integrated experiments based on functional assays (FAs). FAs are intermediary, semi-empirical

51 measures of processes or functions within a specified system that bridge the gap between

52 nanomaterial properties and potential outcomes in complex systems. The three components of a

53 functional assay are standardized protocols for parameter determination and reporting, a

54 theoretical context for parameter application and reference systems. We propose the

55 identification and adoption of reference systems where FAs may be applied to provide parameter

56 estimates for environmental fate and effects models, as well as benchmarks for comparing the

57 results of FAs and experiments conducted in more complex and varied systems. Surface affinity

58 and dissolution rate are identified as two critical FAs for characterizing nanomaterial behavior in

59 a variety of important systems. The use of these FAs to predict bioaccumulation and toxicity for

60 initial and aged nanomaterials is illustrated for the case of silver nanoparticles and C. elegans. 
62 Keywords: dissolution rate, functional assay; nanoEHS, nanomaterial risk assessment; reference

63 systems; risk framework; surface affinity

64 
Introduction

66 The incumbent framework for assessing nanomaterial risk has been one based on an underlying

67 assumption that exposure and hazard can be predicted from physical-chemical properties of

68 engineered nanomaterials (ENMs). This framework is rooted in the view that nanoöbjects

69 essentially resemble chemicals with additional particle-based attributes that must be included

70 among their intrinsic physical-chemical descriptors. The behavior of nanomaterials within this

71 chemical framework is thought to be amenable to description using structure-activity

72 relationships and similar approaches that seek to map intrinsic nanomaterial properties to hazard

73 and exposure. However, with the exception of the trivial case of predicting that nanomaterials

74 made from toxic or highly reactive materials should be toxic, this approach has yielded few

75 actionable guidelines for predicting nanomaterial risk.

Progress is being made in understanding the general factors affecting ENM fate and

77 effects. Important data on the toxicity of a few (primarily) pristine nanomaterials are now

78 available(ICN 2014) across a biological continuum. Factors influencing the environmental fate

79 of nanomaterials are being identified (Alvarez, Colvin et al. 2009, Bone, Colman et al. 2012,

80 Klaine, Koelmans et al. 2012, Lowry, Gregory et al. 2012, Nowack, Ranville et al. 2012, Unrine,

81 Colman et al. 2012, Westerhoff and Nowack 2012, Barton, Therezien et al. 2014), and

82 theoretical models are being developed to predict nanomaterial behavior and impact from these

83 measured behaviors (Scheringer 2008, Praetorius, Scheringer et al. 2012, Dale, Lowry et al.

84 2013, Hendren, Badireddy et al. 2013, Therezien, Thill et al. 2014, Dale, Casman et al. 2015,

85 Quik, de Klein et al. 2015). The growing and necessary focus on the many complex interactions

86 and transformations that impact nanomaterial behavior is being aided by the development of

87 computational methods for data integration (NRC 2012, NRC 2013, NNI 2014). 
Nonetheless, the complexity and breadth of potentially relevant materials (e.g.

nanohybrids used to harvest photons and shuttle electrons in photovoltaics (Saleh, Aich et al. 2015)) and the wide variety of elemental compositions, functions, variations in surface chemistry, and morphology, coupled with a vast number of system-dependent transformations,

92 bioüptake processes, and resulting by-products, makes it challenging to know what nanomaterial

93 properties and environmental variables enable us to describe, predict and therefore control 94 nanomaterial behavior, be it beneficial or harmful.

96 For all the novelty of nanomaterials, the challenges they present from a risk forecasting

97 perspective are similar to those that have been confronted in addressing previous environmental

98 issues ranging from predicting the environmental impacts of synthetic organic chemicals to managing wastewater discharges. In these cases, decision-making was aided by measurements of material behavior in prescribed conditions representing useful simplifications of the environment(s) into which these materials would be introduced. Predictions of oxygen depletion caused by the degradation of complex mixtures of municipal wastes were not obtained from a

103 first principles analysis of the chemical composition of waste water and the composition of 104 microbial populations present in the waste and in surface water (even if these factors were 105 studied later). Rather, functional measurements of oxygen consumption by the bacteria actually 106 present in the waste entering surface waters were obtained and the concept of biochemical 107 oxygen demand (BOD) was introduced. Similarly, environmental fate and bioäccumulation of 108 synthetic organic chemicals (SOCs) was correlated to measurements of the partitioning behavior 109 of compounds between water and a representative organic phase. Trends in chemical partitioning 110 behavior between water and a specific reference organic phase such as octanol $\left(\mathrm{K}_{\mathrm{ow}}\right)$ could be 
111 predicted subsequently from first principles(Schwarzenbach, Gschwend et al. 2005). These 112 examples of what we refer to as functional assays illustrate the utility of making measurements

113 of key variables in complex systems (e.g. oxygen demand) without the need for complete

114 mechanistic understanding of the processes that yield that behavior.

116 As in the cases of BOD and SOCs, the complexity of a system's effects on nanoparticle 117 properties tends to obscure predictive links between intrinsic properties of nanomaterials and 118 their impacts. An expedient alternative to directly linking ENM properties to impacts is to focus 119 on standardized reproducible measurements of relevant nanomaterial behaviors in relevant 120 systems that can inform near-term decision-making. We refer to procedures that produce such 121 measurements as functional assays. To forecast nanomaterial behavior we will need 122 parameterized predictive models, and functional assay measurements provide such parameters as 123 well as the range of values for those parameters. Ideally, functional assays should be pertinent 124 not only for first generation nanomaterials (that roughly conform to the chemical model) but also 125 for more complex nanoöbjects ranging from hybrid materials to active nano-scale devices. In this 126 communication we outline the general features of functional assays for nanoöbjects, focusing on

127 the need to designate reference systems in performing these assays and identifying two key 128 assays that can be easily applied to parameterize models for exposure and, potentially, bioüptake.

\section{Definition of a functional assay}

131 We define functional assays as procedures for quantifying parameters that describe a specific

132 process (or function) occurring within a given (often complex) system. Functional assays provide 133 quantitative information on the rates at which processes occur (e.g., rates of aggregation, 
134 bioäccumulation or precipitation) or indications of performance or final states (e.g., distribution 135 between air/water, living/dead ( $\left.\mathrm{LD}_{50}\right)$, or Young's modulus). Functional assays (FAs) inherently 136 incorporate linkages between nanomaterials properties, nanomaterial function, system

137 characteristics, and the exposure or hazard endpoints of interest; they describe the convergent 138 properties of nanoöbjects and the systems in which they are present (Figure 1b). The parameters 139 measured in these assays may be semi-empirical, expressing the aggregate effects of numerous 140 predicate processes that may in turn be deconstructed to ultimately yield a description of the 141 overall process based on first principles, including knowledge of all component processes as well 142 as the nanomaterial and system properties. FAs therefore bridge individual empirical 143 measurements and long-term generalizable theory in support of near-term nanoEHS guidance 144 and sustainable product development. These assays allow for parameterization of models for 145 transport, transformation, reactivity and effects of nanomaterials.

147 This approach adds a crucial halfway point along the path that is typically discussed with regard to predictive risk assessments for nanomaterials, which often focus on pursuit of quantitative 149 structure-activity relationships (QSARs) and attempts at mapping entire adverse outcome 150 pathways (AOPs). Figures $1 \mathrm{a}$ and $\mathrm{b}$ illustrate (for a fate- and transport-focused example) that the 151 insertion of functional assay measurements as an intermediate, currently measurable predictor of 152 key behaviors that will determine fate and effects of nanomaterials provides an achievable 153 interim goal while also setting the stage for future mechanistic investigation. FAs are particularly 154 needed to predict rates of processes; these measurements group together complex behaviors and 155 imply upstream mechanistic processes that determine their values. FAs do not entirely replace 156 the need for measurements of the nanoparticle properties, system properties and social and 
157 engineered properties; indeed, these measurements and estimates (in particular production

158 volumes and use patterns) will continue to be important for informing exposure estimates and for

159 ultimately linking ENM properties with fate and effects. However, we do not have to

160 mechanistically understand how material and system properties govern transformation processes,

161 for example, in order to quantify the occurrence and rate of such processes in given systems for

162 given materials. Instead, FAs can directly measure the phenomena that cause nanomaterials to

163 form, dissolve, change, move, or impart chemical changes in neighboring constituents.

164 Parameters derived from FAs incorporate complexities in environmental interactions. For

165 example, particle size has been shown to effectively predict Ag NP solubility(Zhang, Yao et al.

166 2011, Ma, Levard et al. 2012, Dobias and Bernier-Latmani 2013), but even small degrees of

167 sulfidation in environmental systems alter solubility(Levard, Reinsch et al. 2011). Measurement

168 of dissolution rate in a prescribed system that is pertinent to the risk evaluation under

169 consideration takes into account the interactions between these two processes as well as other

170 processes that potentially affect dissolution rate (e.g. interaction with NOM)(Gondikas, Morris et

171 al. 2012). These measurements provide immediate and valuable empirical information to predict

172 exposure potential and consequent bioactivity. Later, as data and meta-data are consistently

173 amassed describing the scenarios, materials and systems associated with these functional assay

174 measurements, the data can be interrogated for more fundamental relationships.

175 Standardizing these assays and the conditions and methods by which they are measured offers a

176 path toward amassing data that can support near-term, realistic assessments of potential

177 exposures and hazards.

178 
179 Though many of these concepts are independently well understood, they have yet to be adopted 180 by the nanoEHS community in a systematic way that enables a cohesive and tractable approach

181 to amassing evidence for informing risk-based (or performance-based) decisions on the

182 sustainable development of nanotechnology.

184 The three components of a functional assay are standardized protocols for parameter 185 determination and reporting, reference systems, and a theoretical context for parameter 186 application. Each of these will be addressed here. Though explicit handling of uncertainty is not 187 a topic of this manuscript, it is important to note the existence of inherent uncertainty with regard 188 to identification of the optimal parameters for forecasting behavior, judgment of relevant 189 reference systems, development of measurement and reporting protocols to measure, forecasting 190 exposures and effects of nanomaterials from FA measurements, and the hope that capturing FA 191 measurements with consistent meta-data will ultimately provide the ability to link between 192 nanomaterial and system properties and observed behaviors. Increased awareness and adoption 193 of FA approaches will allow systematic investigation into these uncertainties. 
Figure 1a. Nanoparticle property-rooted approach

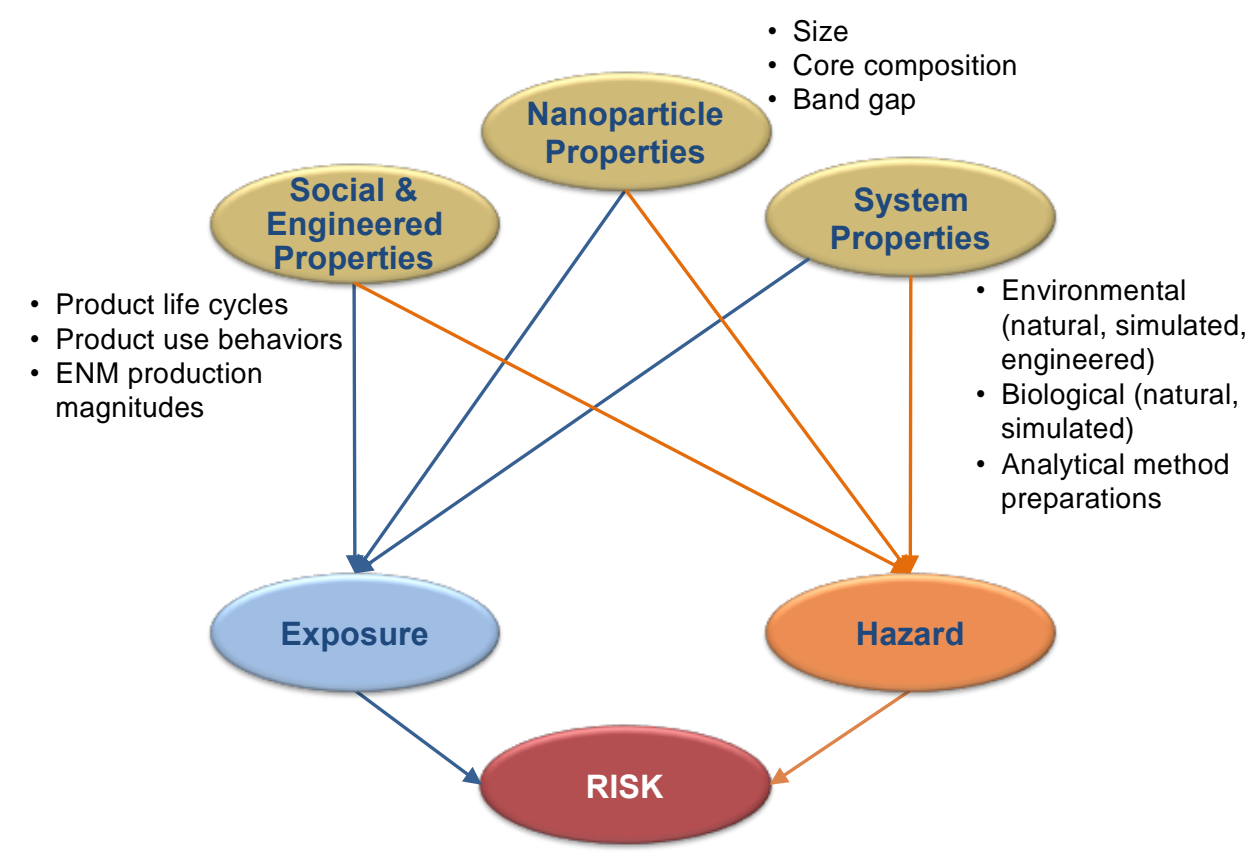

Figure 1b. Functional assay-rooted approach

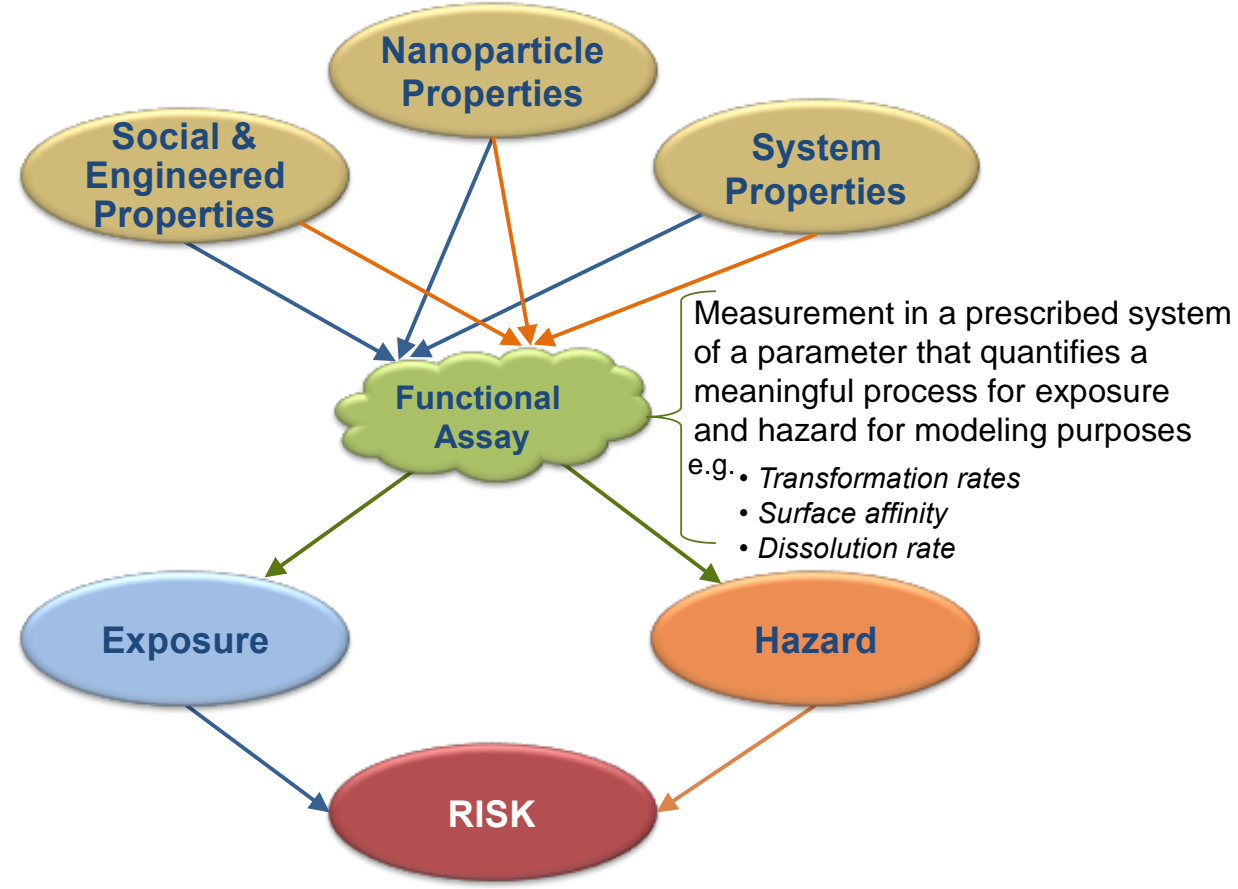


$\underline{\text { Protocols for parameter determination: Intrinsic/extrinsic nanomaterial properties and system }}$

199 properties

200 Many lists of physical-chemical characteristics (e.g., (Thomas, Aguar et al. 2006, Stone, Nowack et al. 2010, OECD 2012)) of nanomaterials found in risk assessment gap analyses or recommended characterization protocols include a mix of properties that are both intrinsic to the material identity as well as those that exist solely as a function of the environmental systems that the nanomaterial passes through. In characterizing nanomaterials in reference systems, there is an increasingly recognized need to separate intrinsic system-independent properties of the materials from those properties that are extrinsic, or system-dependent (2008, Maynard 2009, Kandlikar, Satterfield et al. 2013, Lynch, Weiss et al. 2014), and to explicitly consider system parameters

208 (2008, Klaine, Alvarez et al. 2008, OECD 2012, OECD 2014).

210 argue it means the nanomaterial has a new identity (Figure 2). Other properties of that material

211 are system-dependent, or extrinsic, properties. Making distinctions between intrinsic and

212 extrinsic properties is insufficient without critical information describing the surrounding

213 systems that influenced or induced these extrinsic states of being. The influence of the system on 214 extrinsic nanomaterial properties (Levard, Reinsch et al. 2011) and the role of the physiological 215 properties of the receptor (Judy, Unrine et al. 2011, Unrine, Shoults-Wilson et al. 2012) have 216 been repeatedly demonstrated to be more important to predicting a variety of effects than the 217 nanomaterial properties. Though it could be argued that fundamentally, all nanomaterial 218 properties may eventually change via transformations, one can separate these properties into 219 those that, if they change, would effectively confer a new fundamental identity to the material vs. 220 those that for a material of the same identity could easily change as a function of its 
221 surroundings. Moreover, not all systems are equally likely or pertinent to real world scenarios;

222 hence, the importance of intentionally selected standardized reference systems. All three

223 categories of characterizations in this figure must be included explicitly in designing experiments

224 and compiling datasets to examine nanomaterial behavior: intrinsic properties of a nanomaterial,

225 the surrounding system properties, and the extrinsic properties derived from the nanomaterial

226 existing in the system.

Figure 2. Intrinsic vs. extrinsic (system-dependent) properties; a simple ENM example

\begin{tabular}{|c|c|c|}
\hline $\begin{array}{c}\text { Intrinsic } \\
\text { Properties of a }\end{array}$ & $\begin{array}{c}\text { Extrinsic Properties } \\
\text { or States }\end{array}$ & $\begin{array}{l}\text { Environmental or } \\
\text { Biological Systems }\end{array}$ \\
\hline Nanomaterial & State 1 & In a WWTP Secondary Clarifier \\
\hline $\begin{array}{l}\text { Core composition } \\
\text { Band gap } \\
\text { Particulate diameter }\end{array}$ & $\begin{array}{l}\text { Surface composition } 1 \\
\text { Surface charge } 1 \\
\text { Aggregation state } 1\end{array}$ & $\begin{array}{ll}\text { - } & \text { Roughly neutral } \mathrm{pH} \\
\text { - } & \text { Moderate ionic strength } \\
\text { - } & \text { Oxic conditions } \\
\text { - } & \text { Moderate DOC }\end{array}$ \\
\hline & State 2 & In Fresh Surface Water \\
\hline $\begin{array}{l}\text { Core composition } \\
\text { Band gap } \\
\text { Particulate diameter }\end{array}$ & $\begin{array}{l}\text { Surface composition } 2 \\
\text { Surface charge } 2 \\
\text { Aggregation state } 2\end{array}$ & $\begin{array}{ll}\text { - } & \text { Roughly neutral } \mathrm{pH} \\
\text { - } & \text { Low ionic strength } \\
\text { - } & \text { Oxic conditions }\end{array}$ \\
\hline & State 3 & Subaquatic Freshwater Sediment \\
\hline $\begin{array}{l}\text { Core composition } \\
\text { Band gap } \\
\text { Particulate diameter }\end{array}$ & $\begin{array}{l}\text { Surface composition } 3 \\
\text { Surface charge } 3 \\
\text { Aggregation state } 3\end{array}$ & $\begin{array}{ll}\text { - } & \text { Roughly neutral pH } \\
\text { - } & \text { Low ionic strength } \\
\text { - } & \text { Reducing conditions/sulfidic } \\
\text { - } & \text { Moderate to high DOC }\end{array}$ \\
\hline
\end{tabular}

The need for reference systems

230 While the utility of standardized nanomaterials has been widely recognized as a prerequisite to

231 obtaining comparable data across studies, there has been a surprising lack of standardization of

232 the systems in which nanomaterials should be characterized and evaluated. Reference systems 
233 constitute a critical element of standardized protocols needed for ENM characterization and for

234 meta-data collection associated with these systems, in order to track how system properties

235 impact materials' extrinsic characteristics (Keller, Wang et al. 2010, NRC 2012). Ideally,

236 functional assays determined in contextually relevant reference systems yield quantitative

237 estimates of parameters that can be used in fate model calculations and to assess bioavailability.

238 Rate constants are one such example that enable theoretical mass balance calculations to inform 239 exposure assessment (Dale, Lowry et al. 2013).

241 Because extrinsic properties of nanomaterials may vary as a function of the history of systems

242 that the nanoparticles experience, comparison between experimental results requires contextual

243 information that is provided by a reference system. Throughout the nanoEHS community there is 244 agreement that standardized materials and characterization protocols are needed (Tran,

245 Donaldson et al. 2005, Klaine, Alvarez et al. 2008, EC 2009, DEFRA 2011, NRC 2012, OECD 246 2012, NNI 2014). To date though, even references to clear distinctions between intrinsic and 247 extrinsic nanomaterial characteristics have stopped short of proposing a set of reference systems 248 to correspond to lists of reference nanomaterials (Kandlikar, Ramachandran et al. 2007, 249 Boverhof and David 2010). Some testing protocols include such standardized systems such as 250 EPA Moderately Hard Water (EPA 2007), and many tests are conducted in standard media that 251 may be logical candidates to include in a designated set of reference systems. Ideally, an 252 accepted set of reference systems, or at a minimum, consensus on a set of preferable systems 253 among those already commonly used, will span conditions simulating likely scenarios of interest 254 to regulators and researchers. An associated list of consistent characterization parameters for 
255 various systems aids in comparing measurements made in different systems (e.g., (Keller, Wang 256 et al. 2010, Quadros, Pierson et al. 2013)).

258 Only a finite number of the vast potential combinations of materials and systems can be tested, 259 and not all combinations will be equally relevant to either 1) represent realistic scenarios or 2)

260 allow the prediction of conservative upper bounds of potential effects. Different environmental 261 and biological systems may be useful to evaluate based on their relevance to specific exposure 262 pathways or regulations; considering these reference systems with explicit linkage to their 263 intended risk forecasting context will provide a link in the risk information supply chain that is 264 currently not clear.

266 In defining systems of interest, the opportunity also exists to connect nanoEHS research with 267 industry and nano-application research and development communities. Use of common testing 268 systems could help to develop standards and systems that may be relevant to known exposure 269 scenarios, and to link nano-application evaluation practices with nano-implication evaluation 270 practices as well as regulatory categorization and risk management recommendations.

272 A few examples of commonly used reference systems of environmental relevance that might be 273 considered by the NanoEHS community for adoption are listed in Table 1, along with some 274 representative parameters that define these systems. To enable the goal of comparing 275 nanomaterial behavior across different types of media, more extensive specification and 276 characterization of the media may be required beyond those required for the uses initially 
Table 1: Examples of standardized systems and key associated parameters

\begin{tabular}{|l|l|l|}
\hline $\begin{array}{l}\text { Samples of } \\
\text { system types }\end{array}$ & Examples from literature and current standards & $\begin{array}{l}\text { Parameters specified in current } \\
\text { standard methods }\end{array}$ \\
\hline Seawater & $\begin{array}{l}\text { EPA artificial seawater(EPA 2007) } \\
\text { Santa Barbara seawater(Keller, Wang et al. 2010) } \\
\text { Bodega Bay seawater(Keller, Wang et al. 2010) } \\
\text { Instant Ocean(Park, Tuttle et al. 2013) }\end{array}$ & $\begin{array}{l}\text { Temperature, ionic and mineral } \\
\text { composition }\end{array}$ \\
\hline Freshwater & $\begin{array}{l}\text { EPA reconstituted fresh water (5 variants ranging } \\
\text { from very soft to very hard)(EPA 2007) } \\
\text { Hoagland's hydroponic medium(Hoagland and } \\
\text { Arnon 1950) }\end{array}$ & $\begin{array}{l}\text { pH, mineral composition, hardness, } \\
\text { alkalinity, proportion of mineral water }\end{array}$ \\
\hline $\begin{array}{l}\text { Groundwater } \\
\text { Specified mixture of macro and } \\
\text { micronutrients: N, K, Ca, P, S, Mg, B, } \\
\text { Fe, Mn, Zn, Cu, Mo }\end{array}$ \\
\hline $\begin{array}{l}\text { Synthetic raw } \\
\text { Wastewater }\end{array}$ & $\begin{array}{l}\text { ASTM groundwater sampling guide(ASTM 2013) } \\
\text { (Establishes parameters to be characterized but no } \\
\text { prescribed value ranges) }\end{array}$ & $\begin{array}{l}\text { Flow rate, water level, pH, alkity, } \\
\text { hardness, specific conductance, } \\
\text { turbidity, TSS, specified elemental and } \\
\text { common contaminant concentrations }\end{array}$ \\
\hline $\begin{array}{l}\text { Biosolids/ } \\
\text { Sludge }\end{array}$ & $\begin{array}{l}\text { Synthetic WWTP biosolids(Ma, Levard et al. 2013), } \\
\text { NIST domestic(NIST retrieved July 23, 2014) and } \\
\text { industrial(NIST retrieved July 23, 2014) sludges }\end{array}$ & $\begin{array}{l}\text { Specified elemental concentrations } \\
\text { (mg/kg) }\end{array}$ \\
\hline $\begin{array}{l}\text { Human cell } \\
\text { growth media }\end{array}$ & $\begin{array}{l}\text { e.g., RPMI media(Moore and Woods 1977), } \\
\text { Dulbecco's Modified Eagle Medium } \\
\text { (DMDM)(Ausubel, Brent et al. 2002), etc. }\end{array}$ & $\begin{array}{l}\text { Nutrients required for growth (e.g., } \\
\text { protein, sodium chloride, N,P, } \\
\text { enzymes, growth factors...) }\end{array}$ \\
\hline $\begin{array}{l}\text { Microbial growth } \\
\text { media }\end{array}$ & $\begin{array}{l}\text { e.g., Lysogeny broth(Bertani 2004), MacConkey's } \\
\text { Agar, Mannitol Salt Agar, etc. }\end{array}$ & $\begin{array}{l}\text { Nutrients required for growth (e.g., } \\
\text { protein, agar, sodium chloride, N, P, } \\
\text { lactose...) }\end{array}$ \\
\hline Synthetic saliva & $\begin{array}{l}\text { SAGF medium(Gal, Fovet et al. 2001) } \\
\text { SH, ionic and chemical composition, } \\
\text { ionic strength }\end{array}$ \\
\hline Synthetic sweat & $\begin{array}{l}\text { AATCC, ISO and EN standards (as consolidated in } \\
\text { (Kulthong 2010)) } \\
\text { temperature }\end{array}$ \\
\hline Synthetic urine & S-urine formulation(Mayrovitz and Sims 2001) & pH, chemical composition \\
\hline
\end{tabular}

\section{$\underline{\text { Key functional assays for predicting exposure }}$}

A generic mass balance describing the change in concentration of nanoöbjects of a given size and speciation over time in a system includes source terms (e.g., discharge, biogenesis, or precipitation), reactive transformations (e.g., sulfidation or changes in redox state), reactive envisioned for these media (e.g. ionic strength, $\mathrm{pH}, \mathrm{UV} 254$, conductivity, temperature, flow regime, ionic composition, redox state).
$\mathrm{Fe}, \mathrm{Mn}, \mathrm{Zn}, \mathrm{Cu}, \mathrm{Mo}$ hardness, specific conductance, turbidity, TSS, specified elemental and common contaminant concentrations

Specified elemental concentrations

Nutrients required for growth (e.g., protein, sodium chloride, $N, P$, enzymes, growth factors...

Nutrients required for growth (e.g., protein, agar, sodium chloride, N, P, $\mathrm{pH}$, ionic and chemical composition, onic strength temperature 
287 advection and settling). In addition, nanomaterials may be taken up and depurated by biota.

288 Reactive transformations, transport, reactive losses, bioüptake, and transport are all potentially

289 amenable to description using function assays. The specificity of chemical reactivity and biology

290 present a challenge to identifying generalizable functional assays for reactive transformations

291 and bioüptake, which perhaps may be ultimately addressed through the use of indicator species

292 for bioüptake, or chemical groupings (e.g., redox potential) for reactive transformations.

294 By way of examples, we focus here on functional assays to determine dissolution rate and 295 surface affinity. Assays to determine these parameters may be immediately applied, are easily 296 executed, and ultimately characterize most of the dominant processes dictating the fate of 297 nanomaterial mass in environmental systems. Self-descriptive, the dissolution rate (or leaching 298 rate) characterizes the release of elements contained in nanoöbjects to solution, representing both 299 a loss of nanoparticle mass and a potential source of toxicity. Measurements of dissolution rate in 300 a representative matrix can ultimately be correlated with e.g. bioavailability, toxicity, and 301 persistence in the environment. System properties that, in concert, decrease dissolution rate will 302 all be captured in the measurement without the need to explicitly understand the mechanisms of 303 dissolution. The transport processes of particle aggregation, deposition, and settling are 304 determined in part by the affinity of interacting surfaces. Particle aggregation involves the 305 transport of one particle to the vicinity of another where, if surface affinity is favorable, 306 attachment may occur and aggregates (or agglomerates) may form. Larger aggregates will tend 307 to have faster settling rates. When surface affinity (attachment efficiency) is high, aggregate 308 structure will also tend to be more porous, leading to reduced drag coefficients and faster 309 aggregate settling rates. Similarly, deposition involves the transport of particles to the vicinity of 
310

311

312

\section{Surface Affinity}

314 Surface affinity $(\alpha)$ describes the probability of particle attachment when particles collide with 315

The affinity of a given type of nanoparticle (as manufactured or following modification in the another particle or a stationary "collector" surface. Surface affinity determines the mobility of nanomaterials (and other small particles) in environmental matrices such as porous media (Lecoanet, Bottero et al. 2004) their propensity to heteroäggregate (Brant, Labille et al. 2007) (Therezien, Thill et al. 2014) and, in some cases, the reactivity of nanoparticle aggregates (Jassby, Budarz et al. 2012). Surface affinity is a function of numerous phenomena including van der Waals forces, electrical double-layer interactions, steric interactions, hydration forces, and particle/surface hydrophobicity. It is therefore affected by surface treatments engineered into nanoparticles in the form of charged functional groups or macromolecules. Surface affinity also reflects changes in nanoparticle surface properties due to the adsorption of natural organic matter, or proteins (e.g., protein corona), the conditions of solution chemistry such as $\mathrm{pH}$, ionic strength, specifically sorbing ions, surface heterogeneity, and myriad other factors determined by the system containing the nanoparticle. The value of $\alpha$ is unity when there are no barriers to particle deposition and attachment is favored but may exceed 1 if phenomena at small separation distances draw particles to the surface(Elimelech and O'Melia 1990). All nanoparticles can be characterized with respect to their surface affinity, which will vary as a function of the medium in which nanoparticles are dispersed or embedded.

$$
\text { environment) for a single surface or complex mixture of surfaces (such as a soil, sediment, or }
$$


333 activated sludge) can be determined by introducing the nanoparticles to a suspension of the

334 "background" material of known concentration (Barton, Therezien et al. 2014). Following a

335 programmed period of heteroäggregation, background particles are separated, typically by

336 settling, from the suspension. The surface affinity is determined from measurements of the

337 concentration of nanoparticles remaining in suspension, $n$, in the context of a simplified

338 Smoluchowski equation ((Smoluchowski 1917)) describing heteroäggregation between

339 nanoparticles and background particles of concentration B. The heteroäggregation rate is given

340 as the product of the second order collision rate constant, $\beta(n, B)$, the concentration of un-

341 heteroäggegated nanoparticles, $n$, the concentration of background particles, B (which remains

342 constant), and the affinity coefficient between nano- and background- particles, $\alpha_{\mathrm{NB}}$. A reverse

343 reaction due to break-up can be assumed to be proportional (by the rate constant $\mathrm{k}_{\mathrm{B}}$ ) to the

344 number of nanoparticles that have heteroäggregated $\left(\mathrm{n}_{0}-\mathrm{n}\right)$ such that:

$$
\frac{d n}{d t}=-\alpha_{N B} \beta(n, B) n B+k_{B}\left(n_{o}-n\right)
$$

Initially, breakup can be assumed to be negligible and the rate equations describing aggregation

347 between nanoparticles and background particles can be solved to yield a predicted linear

348 relations ship between the natural logarithm of $\mathrm{n}_{0} / \mathrm{n}$ and mixing time:

$$
\ln \left(\frac{n_{0}}{n}\right)=\alpha_{N B} \beta(n, B) B t
$$

350 Linear regression of the linear portion of the graph will provide a trend line where the slope of

351 the line will be $\alpha \beta B$. Dividing by the number concentration of the background and a calculated

352 collision frequency yields a value for $\alpha_{N B}$. Alternatively, $\alpha_{N B}$ can be obtained by normalizing 
353 values of $\alpha \beta B$ to the slope obtained when conditions are manipulated to yield a surface affinity

354 that approaches unity (such as high ionic strength in the absence of steric stabilization).

355 This method has been applied to determine surface affinities for a series of metal and metal oxide

356 NPs heteroäggregated with activated sludge (Barton, Therezien et al. 2014). We have further

357 demonstrated that the trends in surface affinity for idealized reference systems of nanoparticles

358 and glass beads follow well-established behaviors with respect to $\mathrm{pH}$, ionic strength, and the 359 concentration of natural organic matter known for colloidal systems.

361 Dissolution rate, $\mathrm{k}_{\underline{\mathrm{d}}}\left(\mathrm{md}^{-1}\right)$

362 Some NPs will undergo dissolution in the matrix that they are in (e.g. lake water, lung fluid, 363 sediment porewater). Dissolution rate describes the speed at which the NP forms a solution in a 364 given solvent, and may be critical to predicting both the persistence and fate of and hazard posed 365 by soluble NPs. In many cases it is the release of metal cations from the NPs that promotes 366 bioavailability and toxicity, though this may not always be the case (e.g. filter feeders or other 367 organisms that ingest particles)(Luoma 2008, Fabrega, Luoma et al. 2011). Many environmental 368 factors can influence dissolution rate, e.g. redox conditions, presence of sulfide or other strong 369 ligands for the NPs. Despite the complexity surrounding the interactions of various ligands and 370 redox conditions, a simple assay in relevant environmental media can be used to measure the 371 dissolution rate. We propose that developing a class of functional assays to measure dissolution 372 rates for particular systems of interest could enable forecasting of their fate and effects in the 373 environment. 
375 It is important to distinguish between dissolution rate and solubility in a functional assay. The

376 former is a reasonable surrogate for the delivery rate of metal cations under dynamic conditions

377 where the released ions are carried away from the particles, while the latter is an equilibrium

378 measurement that does not provide any rate information. Under most conditions, NPs will be

379 present at low concentration and in a dynamic environment such that equilibrium is not

380 established, so the rate of dissolution is the important parameter to consider. Thus, a functional

381 assay should be designed to provide an estimate of the dissolution rate under representative

382 environments.

383

384 Three types of dissolution rate functional assays will likely be necessary. The first is dissolution 385 rate measured in a liquid, e.g. freshwater, seawater, or porewater environments. The chemical 386 composition of the solution (e.g. presence/absence of ligands) and redox conditions must reflect 387 the environmental conditions of the environment being considered; the basic measurement will 388 be the same for each type of matrix sample. The dissolution rate can be measured at a low NP 389 to solution ratio, and over a time period long enough to assess the rate of dissolution and any 390 changes in rate that may result. The important consideration for most NPs would be to ignore 391 transient behavior at the beginning of the measurement and focus on the longer-term dissolution 392 rate. The transient behavior could include rapid initial dissolution due to release of adsorbed 393 ions (Dobias and Bernier-Latmani 2013), or slower dissolution due to a protective coating 394 (Sotiriou, Watson et al. 2014). However, if these are relatively short-term effects (e.g. <1d), they 395 may not be important when considering the long-term dissolution behavior of the material. 
There are many potential functional assays to measure dissolution rate. One can follow the procedures used by many researchers: addition of NPs to the solution, brief sonication as needed, mixing on an orbital shaker for the duration of the test to minimize mass transfer resistance, and periodic measurements using centrifugal filtration with a 3 or $10 \mathrm{kDa}$ filter to separate ions from NPs (Liu, Sonshine et al. 2010, Ma, Levard et al. 2012). Testing may eventually lead to the need for a larger MWCO filter that includes metal-macromolecule complexes in the "filterable/bioavailable fraction" to be measured in the test (Ma, Stegemeier et al. 2014, Li, Hu et al. 2015). These are simple batch tests adaptable to any type of solution that best represents the system of interest (e.g. freshwater, sediment porewater, wastewater treatment activated sludge), and can eventually be automated. One potential problem with this batch approach is that redox conditions may change during the test, or the ligands present in the solution that promote dissolution may become depleted. This can likely be avoided by using a low concentration of NPs. However, more complex flow-based systems may also be used for this purpose. For example, Kent et al., 2012 used a flow through cell containing deposited NPs for such measurements. In their experiments, the concentration of ion was monitored in the cell outlet and the inlet conditions (ligands, redox, $\mathrm{pH}$, flow, etc.) were easily controlled (Kent and Vikesland 2012). One could imagine a standard method for depositing particles in a flow cell would enable monitoring of dissolution rates under a variety of conditions. It would also be possible to expose deposited NPs to representative conditions, e.g. WWTP activated sludge, prior to making dissolution measurements. The flow through system would have better control, but would be more complicated to implement. For comparability with other data, all dissolution rate data should be reported as a surface recession rate, $\mathrm{m}^{3}$ reacted per $\mathrm{m}^{2}$ of surface area per $\mathrm{d}$ (Liu, Sonshine et al. 2010). 
421 A second dissolution assay is needed for nanomaterials in soils and sediments. In soils, moisture

422 content, heteroäggregation, $\mathrm{pH}$, and organic constituents can all affect the solubility of NPs in

423 soils. Soil systems may range from fully water saturated (subaquatic sediment) to partially

424 saturated soils. Sorption-desorption and leaching studies (e.g. OECD test guidelines 106 and

425312 ) are not necessarily suitable to determine the solubility of NPs in soils. A functional assay

426 that may be appropriate for soils is a measurement using DGT (Harper, Davison et al. 1998,

427 Ernstberger, Zhang et al. 2005). DGT devices can be emplaced in soils, recovered, and the mass

428 of metal released from the NPs is measured in the gel. Dialysis coatings placed over the gel

429 surface can be used to eliminate the uptake of NPs (Sekine, Brunetti et al. 2015). Simple models

430 and an equilibrium measurement between the metal of interest and the soil are used to calculate

431 the "delivery rate" of metal ions derived from the NPs (Harper, Davison et al. 2000, Ernstberger,

432 Davison et al. 2002). This rate of delivery of metal may ultimately be correlated to the

433 bioavailability and/or toxicity (Tandy, Mundus et al. 2011). These devices are versatile and can

434 be used under a range of moisture contents and redox conditions. A simpler method may be a

435 modification of TG 106 where extracted pore water is recovered, filtered with an appropriate

436 MWCO filter, and the soluble fraction determined at a given time point (Whitley, Levard et al.

437 2013). Monitoring the increase in soluble fraction with time can give you the required estimate

438 of dissolution rate. Care must be taken here to avoid reaching the solubility limit of the NPs in

439 the porewater. Careful choice of reference soils and soil conditions is also needed to best

440 represent the range of soil conditions ( $\mathrm{pH}$, organic content, etc.) that may be encountered. A

441 dissolution functional assay may be needed for measuring the dissolution rate of NPs that are

442 incorporated into commercial or consumer products. The encapsulation of NMs into polymeric 
443 matrices, or adhered to surfaces, can alter their dissolution rate. There is a large range of

444 polymeric matrices and potential methods to encapsulate those NPs. However, a FA that

445 measures the rate of leaching of NPs and ions from those products may be sufficient to predict

446 their toxicity potential from metal and/or NP exposures downstream of the devices. Once again,

447 careful choice of the matrix (e.g. artificial saliva, fresh water, saltwater, landfill leachate) will be

448 required to provide results that are predictive of that product in the matrix of interest and via the 449 exposure route of interest.

450

451 Conceptual demonstration of the role of $\alpha$ and dissolution rate in forecasting nanomaterial fate,

452 effects, and relative risk:

453 Figure 3 reprises the graphical abstract for this paper to present a hypothetical example of how 454 two functional assays, surface affinity $(\alpha)$ and dissolution rate $\left(\underline{k}_{d}\right)$, predict relative risks for two 455 different use and exposure scenarios for PVP-coated Ag NPs. 


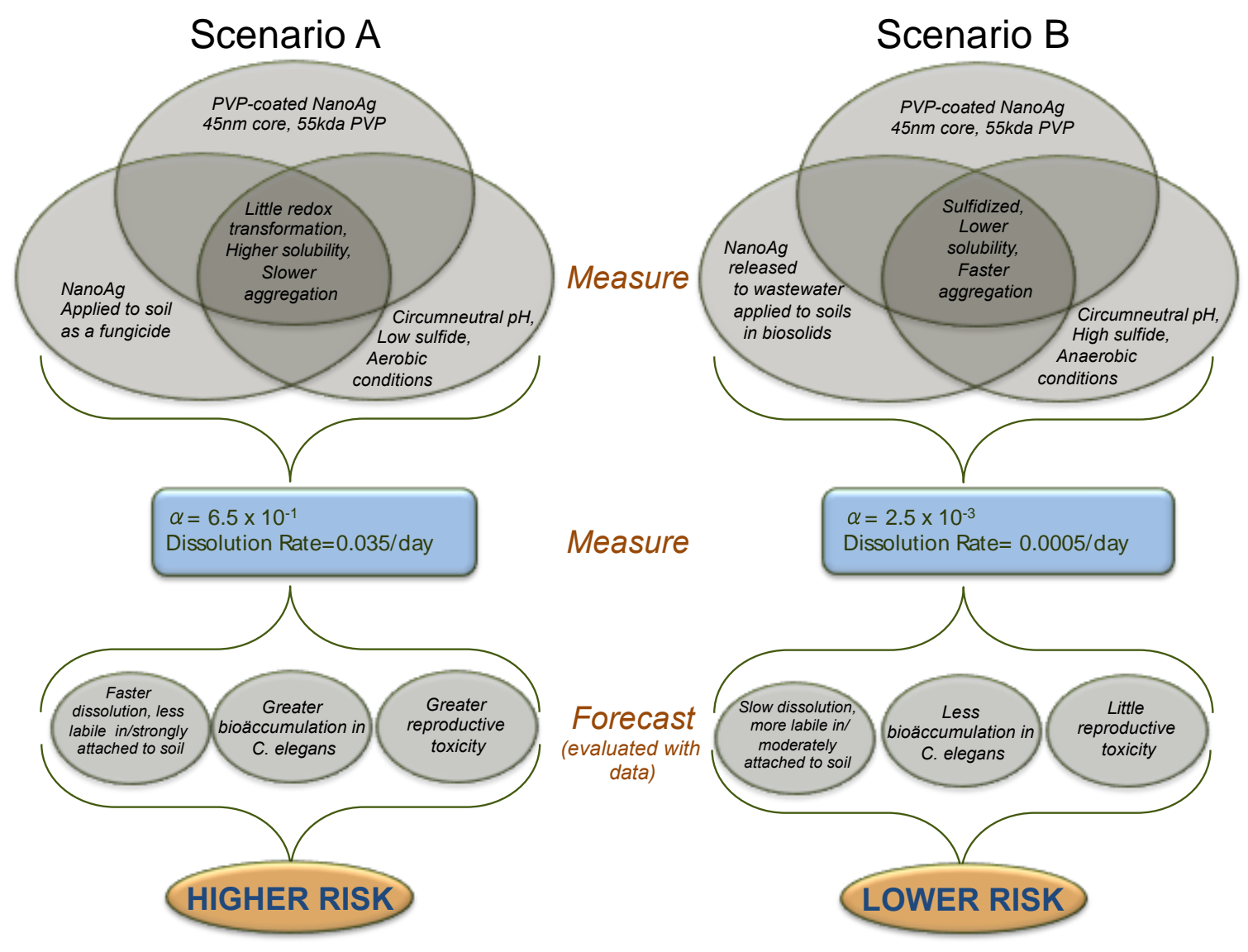

Figure 3: Hypothetical example of applying 2 functional assays to forecast Ag NP behavior under different scenarios.

458 Scenario A is based on the direct application of Ag NPs to soil as a fungicide, which has been

459 described as a possible use scenario (Lamsal, Kim et al. 2011, Sawinska, Khachatryan et al.

460 2014).

461 In this scenario the particles are exposed to aerobic conditions with low sulfide concentrations

462 that would result in limited sulfidation but relatively higher oxidative dissolution (Levard,

463 Reinsch et al. 2011). Scenario B is based on the release of Ag NPs to wastewater from

464 antimicrobial textiles and subsequent processing in a wastewater treatment plant where $\mathrm{Ag}$

465 particles are typically extensively if not completely sulfidized (WWTP) (Kaegi, Voegelin et al. 
2011, Lombi 2013, Ma, Levard et al. 2013, Ma, Stegemeier et al. 2014). In this scenario, surface affinity $(\alpha)$ of sulfidized PVP-coated Ag NPs to glass beads in a packed column is approximately two orders of magnitude smaller than that for unsulfidized PVP-coated Ag NPs (O'Brien unpublished data; see Supplemental Materials). Dissolution rate in moderately hard water (as a proxy for soil pore water) is two also orders of magnitude lower for the sulfidized PVP coated Ag NPs compared with the unsulfidized particles (Starnes, Unrine et al. 2015). Qualitatively, these differences in dissolution rate and $\alpha$ are consistent with our observations that the sulfidized Ag NPs resulted in decreased Ag bioäccumulation and toxicity in a model soil invertebrate, Caenorhabditis elegans, than for unsulfidized PVP-coated Ag NPs (Starnes, Unrine et al. 2015). Ag NPs attachment to surfaces is favored and, once attached, these unsulfidized NPs more readily deliver dissolved $\mathrm{Ag}$ to the organism. The differences in FA values would have also predicted decreased attachment to soil solids, and increased mobility and decreased dissolution in soil for sulfidized Ag NPs in a sandy loam as compared to unsulfidized particles, as has been observed over the short term by Whitley et al. (Whitley, Levard et al. 2013). In terms of Ag mobility, bioavailability, and toxicity in soil invertebrates, these two functional assays would have been predictive of the higher relative risk seen in Scenario A as compared to Scenario B. While based on experimental data, this is a hypothetical example with embedded assumptions (e.g. that the loading of $\mathrm{Ag}$ is similar in both scenarios. The power of these functional assays would be increased if parameters measured in controlled reference systems (e.g., glass beads) can be shown to be predictive of measurements obtained in more complex systems of variable pore water, soil composition and ecological receptor species. 


\section{Conclusions}

490 Orienting risk assessment research around the identification of functional assays and the 491 systematic generation of measurements for those functional parameters that include consistent meta-data on both material AND system characterizations will allow the nanoEHS community to

493 work the problems backwards, holding endpoint responses constant and querying which

494 functional assays appear to be most predictive, and ultimately to identify which material and 495 system parameters matter - or do not matter - in affecting outcomes of interest. This focus is 496 important in searching for functional assays that ideally allow for generalization between 497 systems. The measurements might be adapted for distinct systems, but we should be searching 498 for those parameters that, like rate constants for transformation reactions or equilibrium 499 constants like $\mathrm{K}_{\mathrm{ow}}$ for neutral organic compounds, could be applied to predict material fate in many different systems. Systematic approaches must be developed as a community for making

501 the types of critical choices presented in our example FAs of surface affinity and dissolution, 502 such as the relevant environmental matrices in which they are measured and the associated 503 characteristics that must be reported. This will be equally important for reducing uncertainties of 504 FA applications as well as for appropriate interpretation of FA results in providing guidance (e.g. 505 categorizing nanomaterials for tiered testing strategies, determining subsequent experimental 506 designs).

507 To summarize, we underscore the following proposals of factors to be taken into account in 508 advancing nanoEHS work to achieve near-term predictions of nanomaterial risks:

509 1. Nanomaterial characterization that clearly distinguishes between intrinsic and extrinsic, $510 \quad$ i.e. system-dependent, characteristics. 
2. Adoption of reference systems - and associated minimum criteria for characterizing these systems - to represent environmental and biological systems that are expected to be of particular importance across the value chain and life cycle of nanomaterials and their transformation by-products. Such reference systems will allow the community to amass interoperable data across a relevant spectrum of system properties.

3. Identification of key functional assays and associated parameters that will improve our power to predict nanomaterial fate and impacts, and generation of data on these parameters (with consistent metadata as described above) in systems of interest to forecast outcomes of interest. Benchmark data generated by applying these assays to reference nanomaterials and reference systems can then be compared with results obtained from more complex systems. Serving as a link between completely empirical observations and first principles mechanistic theories, FA parameters may allow us to group material-system combinations that impart similar results.

Basic science is unfolding as we better understand matter on the nano scale. This scale itself has always existed; our ability to manipulate, characterize, and harness the novel advantageous properties is new. We are learning what mechanisms govern the behavior of engineered, naturally occurring, and incidental nanomaterials, and their subsequent interactions and impacts on the environment and health. Functional assays provide a systematic path forward that will be critical for planning research rationally, collecting the resulting data coherently to allow for postanalysis, and aligning databases with the targeted analyses they will support. The integrated research strategy enabled by community-wide adoption of these calls, both in terms of exploring applications and predicting implications of ENMs, is in line with the visions put forth by both the National Nanotechnology Initiative (NNI 2014) and the National Research Council (NRC 2013) 
534 in the US and the NanoSafety Cluster in the EU (Savolainen, Backman et al. 2013).

535 Distinguishing and consistently capturing material and environmental system data in conjunction

536 with generating functional assay parameters will help realize the promise of nanoEHS research

537 to inform near-term decisions, while laying the foundation for fundamental first principles

538 discovery.

540 Acknowledgments:

541 The authors are very thankful to Dr. Niall O'Brien for providing data on $\alpha$ to support this work.

542 We also gratefully acknowledge the work of the more than 130 researchers within the network of 543 the Center for Environmental Implications of NanoTechnology, whose translational efforts in 544 terms of disciplines, experimental scale and complexity have clarified and illuminated these 545 concepts. This material is based upon work supported by the National Science Foundation 546 (NSF) and the Environmental Protection Agency (EPA) under NSF Cooperative Agreement EF5470830093 and DBI-1266252, Center for the Environmental Implications of NanoTechnology 548 (CEINT). Any opinions, findings, conclusions or recommendations expressed in this material are 549 those of the author(s) and do not necessarily reflect the views of the NSF or the EPA. This work 550 has not been subjected to EPA review and no official endorsement should be inferred. 


\section{References:}

555

556

557

558

559

560

561

562

563

564

565

566

567

568

569

570

571

572

573

574

575

576

577

578

579

580

581

582

583

584

585

586

587

588

589

590

591

592

593

594

595

596

597

598

599

(2008). MINChar Physicochemical Parameters List: Recommended Minimum Physical and Chemical Parameters for Characterizing Nanomaterials on Toxicology Studies Woodrow Wilson International Center.

Alvarez, P. J. J., V. Colvin, J. Lead and V. Stone (2009). "Research Priorities to Advance Eco-Responsible Nanotechnology." ACS Nano 3(7): 1616-1619. ASTM (2013). ASTM D4448 - 01(2013): Standard Guide for Sampling Ground-Water Monitoring Wells. ASTM Book of Standards West Conshohocken, PA, ASTM International. 04.09

Ausubel, F. M., R. Brent, R. E. Kingston, D. D. Moore, J. Seidman, J. A. Smith and K. Struhl (2002). Short protocols in molecular biology: a compendium of methods from current protocols in molecular biology, Wiley New York.

Barton, L. E., M. Therezien, M. Auffan, J. Y. Bottero and M. R. Wiesner (2014). "Theory and Methodology for Determining Nanoparticle Affinity for Heteroaggregation in Environmental Matrices Using Batch Measurements." Environmental Engineering Science 31(7): 421-427.

Bertani, G. (2004). "Lysogeny at Mid-Twentieth Century: P1, P2 and Other Experimental Systems." Journal of Bacteriology 186(3): 595-600.

Boeije, G., R. Corstanje, A. Rottiers and D. Schowanek (1999). "Adaptation of the CAS test system and synthetic sewage for biological nutrient removal: Part I: development of a new synthetic sewage." Chemosphere 38(4): 699-709.

Bone, A. J., B. P. Colman, A. P. Gondikas, K. Newton, K. H. Harrold, J. M. Unrine, S. J. Klaine, C. W. Matson and R. T. Di Giulio (2012). "Biotic and abiotic interactions in aquatic microcosms determine fate and toxicity of Ag nanoparticles: Part 2 -Toxicity and chemical speciation." Environmental Science and Technology 46(13): 6925-6933.

Boverhof, D. and R. David (2010). "Nanomaterial characterization: considerations and needs for hazard assessment and safety evaluation." Analytical and Bioanalytical Chemistry 396(3): 953-961.

Brant, J. A., J. Labille, J. Y. Bottero and M. R. Wiesner (2007). Nanoparticle transport, aggregation, and deposition. Environmental Nanotechnology. M. R. Wiesner and J. Y. Bottero. New York, NY, McGraw-Hill. Dale, A. L., E. A. Casman, G. V. Lowry, J. R. Lead, E. Viparelli and M. Baalousha (2015). "Modeling Nanomaterial Environmental Fate in Aquatic Systems." Environmental Science \& Technology 49(5): 2587-2593.

Dale, A. L., G. V. Lowry and E. A. Casman (2013). "Modeling Nanosilver Transformations in Freshwater Sediments." Environmental Science \& Technology 47(22): 12920-12928.

DEFRA (2011). Characterising the Potential Risks posed by Engineered Nanoparticles: A Second UK Government Research Report. F. a. R. A. Department for Environment. London, UK, Nobel House. Dobias, J. and R. Bernier-Latmani (2013). "Silver Release from Silver Nanoparticles in Natural Waters." Environmental Science \& Technology 47(9): 4140-4146.

EC (2009). Work Programme 2010 Cooperation Theme 4: Nanosciences, Nanotechnologies, Materials and New Production Technologies, NMP, European Commission.

Elimelech, M. and C. R. O'Melia (1990). "Effect of particle size on collision efficiency in the deposition of brownian particles with electrostatic energy barriers." Langmuir 6: 1153-1163.

EPA (2007). Wet Methods and Manuals. Section 7: Dilution water: 31-35.

Ernstberger, H., W. Davison, H. Zhang, A. Tye and S. Young (2002). "Measurement and dynamic modeling of trace metal mobilization in soils using DGT and DIFS." Environmental science \& technology 36(3): 349-354.

Ernstberger, H., H. Zhang, A. Tye, S. Young and W. Davison (2005). "Desorption kinetics of Cd, Zn, and Ni measured in soils by DGT." Environmental science \& technology 39(6): 1591-1597. 
Fabrega, J., S. N. Luoma, C. R. Tyler, T. S. Galloway and J. R. Lead (2011). "Silver nanoparticles: behaviour and effects in the aquatic environment." Environment international 37(2): 517-531.

Gal, J.-Y., Y. Fovet and M. Adib-Yadzi (2001). "About a synthetic saliva for in vitro studies." Talanta 53(6): 1103-1115.

Gondikas, A. P., A. Morris, B. C. Reinsch, S. M. Marinakos, G. V. Lowry and H. Hsu-Kim (2012). "Cysteineinduced modifications of zero-valent silver nanomaterials: Implications for particle surface chemistry, aggregation, dissolution, and silver speciation." Environmental Science \& Technology 46(13): 7037-7045. Harper, M. P., W. Davison and W. Tych (2000). "DIFS-a modelling and simulation tool for DGT induced trace metal remobilisation in sediments and soils." Environmental Modelling \& Software 15(1): 55-66. Harper, M. P., W. Davison, H. Zhang and W. Tych (1998). "Kinetics of metal exchange between solids and solutions in sediments and soils interpreted from DGT measured fluxes." Geochimica et Cosmochimica Acta 62(16): 2757-2770.

Hendren, C. O., A. R. Badireddy, E. Casman and M. R. Wiesner (2013). "Modeling nanomaterial fate in wastewater treatment: Monte Carlo simulation of silver nanoparticles (nano-Ag)." Science of the Total Environment 449: 418-425.

Hoagland, D. R. and D. I. Arnon (1950). The water-culture method for growing plants without soil. College of Agriculture, University of California, Berkeley, CA. ICN (2014). Time Progressive Distribution Analysis of NanoEHS Research: Nano EHS Database Analysis Tool. International Council on Nanotechnology.

Jassby, D., J. F. Budarz and M. Wiesner (2012). "Impact of Aggregate Size and Structure on the Photocatalytic Properties of TiO2 and ZnO Nanoparticles." Environmental Science \& Technology 46(13): 6934-6941.

Judy, J. D., J. M. Unrine and P. M. Bertsch (2011). "Evidence for Biomagnification of Gold Nanoparticles within a Terrestrial Food Chain." Environmental Science and Technology 45(2): 776-781.

Kaegi, R., A. Voegelin, B. Sinnet, S. Zuleeg, H. Hagendorfer, M. Burkhardt and H. Siegrist (2011). "Behavior of Metallic Silver Nanoparticles in a Pilot Wastewater Treatment Plant." Environmental Science \& Technology 45(9): 3902-3908.

Kandlikar, M., G. Ramachandran, A. D. Maynard, B. Murdock and W. A. Toscano (2007). "Health risk assessment for nanoparticles: a case for using expert judgement." Journal of Nanoparticle Research 9: 137-156.

Kandlikar, M., T. Satterfield, C. Beaudrie, R. Gregory, G. Long and T. Wilson (2013). Nanotechnology Risk Screening using a Structured Decision Making (SDM) Approach: A summary of results from a nanotechnology experts workshop.

Keller, A. A., H. Wang, D. Zhou, H. S. Lenihan, G. Cherr, B. J. Cardinale, R. Miller and Z. Ji (2010). "Stability and Aggregation of Metal Oxide Nanoparticles in Natural Aqueous Matrices." Environmental Science \& Technology 44(6): 1962-1967.

Kent, R. D. and P. J. Vikesland (2012). "Controlled evaluation of silver nanoparticle dissolution using atomic force microscopy." Envirionmental Science and Technology 46(13): 6977-6984. Klaine, S. J., P. J. J. Alvarez, G. E. Batley, T. F. Fernandes, R. D. Handy, D. Y. Lyon, S. Mahendra, M. J. McLaughlin and J. R. Lead (2008). "Nanomaterials in the environment: Behavior, fate, bioavailability, and effects." Environmental Toxicology and Chemistry 27(9): 1825-1851.

Klaine, S. J., A. A. Koelmans, N. Horne, S. Carley, R. D. Handy, L. Kapustka, B. Nowack and F. von der Kammer (2012). "Paradigms to assess the environmental impact of manufactured nanomaterials." Environmental Toxicology and Chemistry 31(1): 3-14.

Kulthong, K. S., S.; Boonpavanitchakul, K.; Kangwansupamonkon, W.; Maniratanachote, R. (2010). "Determination of silver nanoparticle release from antibacterial fabrics into artificial sweat." Part. Fibre Toxicol. 7. 
Lamsal, K., S. W. Kim, J. H. Jung, Y. S. Kim, K. S. Kim and Y. S. Lee (2011). "Application of Silver Nanoparticles for the Control of Colletotrichum Species In Vitro and Pepper Anthracnose Disease in Field." Mycobiology 39(3): 194-199.

Lecoanet, H. F., J.-Y. Bottero and M. R. Wiesner (2004). "Laboratory assessment of the mobility of nanomaterials in porous media." Environmental Science \& Technology 38: 5164-5169. Levard, C., B. C. Reinsch, F. M. Michel, C. Oumahi, G. V. Lowry and G. E. Brown (2011). "Sulfidation processes of PVP-coated silver nanoparticles in aqueous solution: impact on dissolution rate." Environmental Science \& Technology 45(12): 5260-5266. Li, L., L. H. Hu, Q. Z. Zhou, C. Huang, Y. Wang, C. Sun and G. Jiang (2015). "Sulfidation as a Natural Antidote to Metallic Nanoparticles Is Overestimated: CuO Sulfidation Yields CuS Nanoparticles with Increased Toxicity in Medaka (Oryzias latipes) Embryos." Environmental Science \& Technology 49(4): 2486-2495.

Liu, J., D. A. Sonshine, S. Shervani and R. H. Hurt (2010). "Controlled release of biologically active silver from nanosilver surfaces." ACS Nano 4(11): 6903-6913.

Lombi, E. D., E; Taheri, S; Tavakkoli, E; Jamting, AK; McCluer, S; Naidu, R; Miller, BW; Scheckel, KG; Vasilev, K (2013). "Transformation of four silver/silver chloride nanoparticles during anaerobic treatment of wastewater and post-processing of sewage sludge." Environmental Pollution 176: 193-197. Lowry, G. V., K. B. Gregory, S. C. Apte and J. R. Lead (2012). "Transformations of Nanomaterials in the Environment." Environmental Science and Technology 46(13): 6893-6899. Luoma, S. N. (2008). "Silver nanotechnologies and the environment." The Project on Emerging Nanotechnologies Report 15.

Lynch, I., C. Weiss and E. Valsami-Jones (2014). "A strategy for grouping nanomaterials based on key physico-chemical descriptors as a basis for safer-by-design NMs." Nano Today 9: 266-270. Ma, R., C. Levard, J. D. Judy, J. M. Unrine, M. Durenkamp, B. Martin, B. Jefferson and G. V. Lowry (2013). "Fate of zinc oxide and silver nanoparticles in a pilot wastewater treatment plant and in processed biosolids." Environmental science \& technology 48(1): 104-112.

Ma, R., C. Levard, S. M. Marinakos, Y. Cheng, J. Liu, F. M. Michel, G. E. Brown Jr. and G. V. Lowry (2012). "Size-Controlled Dissolution of Organic-Coated Silver Nanoparticles." Environmental Science \& Technology 46(2): 752-759.

Ma, R., C. Levard, F. M. Michel, G. E. Brown and G. V. Lowry (2013). "Sulfidation Mechanism for Zinc Oxide Nanoparticles and the Effect of Sulfidation on Their Solubility." Environmental Science \& Technology. Ma, R., J. Stegemeier, C. Levard, J. G. Dale, C. W. Noack, T. Yang, G. E. Brown and G. V. Lowry (2014). "Sulfidation of copper oxide nanoparticles and properties of resulting copper sulfide." Environmental Science: Nano 1(4): 347-357.

Maynard, A. (2009). "Living in a post-chemistry world - the regulatory challenges of emerging nanotechnologies." 2020 Science: A clear perspective on emerging science and technology http://2020science.org/2009/09/11/living-in-a-post-chemistry-world-the-regulatory-challenges-ofemerging-nanotechnologies/.

Mayrovitz, H. N. and N. Sims (2001). "Biophysical Effects of Water and Synthetic Urine on Skin." Advances in Skin \& Wound Care 14(6): 302-308.

Moore, G. E. and L. K. Woods (1977). "Culture media for human cells-RPMI 1603, RPMI 1634, RPMI 1640 and GEM 1717." TCA manual / Tissue Culture Association 3(1): 503-509. NIST (retrieved July 23, 2014). SRM 2781 - Domestic Sludge. NIST Chemistry WebBook, NIST Standard Reference Database Number 69 P. J. Linstrom and W. G. Mallard. Gaithersburg MD, 20899, http://webbook.nist.gov. 
NIST (retrieved July 23, 2014). SRM 2782 - Industrial Sludge. NIST Chemistry WebBook, NIST Standard Reference Database Number 69 P. J. Linstrom and W. G. Mallard. Gaithersburg MD, 20899, http://webbook.nist.gov. NNI (2014). National Nanotechnology Initiative Strategic Plan. E. National Science and Technology Council Committee on Technology; Subcommittee on Nanoscale Science, and Technology. Nowack, B., J. F. Ranville, S. Diamond, J. A. Gallego-Urrea, C. Metcalfe, J. Rose, N. Horne, A. A. Koelmans and S. J. Klaine (2012). "Potential scenarios for nanomaterial release and subsequent alteration in the environment." Environmental Toxicology and Chemistry 31(1): 50-59. NRC (2012). A Research Strategy for the Environmental, Health and Safety Aspects of Engineered Nanomaterials. N. R. Council. Washington, D.C., The National Academies Press. NRC (2013). Research Progress on Environmental, Health and Safety Aspects of Engineered Nanomaterials. N. R. Council. Washington D.C., The National Academies Press. OECD (2012). Series on the Safety of Manufactured Nanomaterials: Guidance on Sample Preparation and Dosimetry for the Safety Testing of Manufactured Nanomaterials. O. f. E. C.-o. a. Development. No. 36: 24-37. OECD (2012). Series on the Safety of Manufactured Nanomaterials: Important Issues on Risk Assessment of Manufactured Nanomaterials. O. f. E. C.-o. a. Development. No. 33: 24-27.

OECD (2014). Ecotoxicology and environmental fate of manufactured nanomaterials: test guidelines $O \mathrm{f}$. E. C.-o. a. Development. No. 40: 19.

Park, K., G. Tuttle, F. Sinche and S. Harper (2013). "Stability of citrate-capped silver nanoparticles in exposure media and their effects on the development of embryonic zebrafish (Danio rerio)." Archives of Pharmacal Research 36(1): 125-133.

Praetorius, A., M. Scheringer and K. Hungerbühler (2012). "Development of Environmental Fate Models for Engineered Nanoparticles-A Case Study of TiO2 Nanoparticles in the Rhine River." Environmental Science \& Technology 46(12): 6705-6713.

Quadros, M. E., R. Pierson, N. S. Tulve, R. Willis, K. Rogers, T. A. Thomas and L. C. Marr (2013). "Release of Silver from Nanotechnology-Based Consumer Products for Children." Environmental Science \& Technology 47(15): 8894-8901.

Quik, J. T. K., J. J. M. de Klein and A. A. Koelmans (2015). "Spatially explicit fate modelling of nanomaterials in natural waters." Water Research 80(0): 200-208. Saleh, N. B., N. Aich, J. Plazas-Tuttle, J. R. Lead and G. V. Lowry (2015). "Research strategy to determine when novel nanohybrids pose unique environmental risks." Environmental Science: Nano 2(1): 11-18. Savolainen, K., U. Backman, D. Brouwer, B. Fadeel, T. Fernandes, T. Kuhlbusch, R. Landsiedel, I. Lynch and L. Pylkkänen (2013). "Nanosafety in Europe 2015-2025: towards safe and sustainable nanomaterials and nanotechnology innovations." Finnish Institute of Occupational Health, Helsinki.

Sawinska, Z., K. Khachatryan, L. Sobiech, R. Idziak, T. Kosiada and G. Skrzypczak (2014). "Use of silver nanoparticles as a fungicide." Przemysl Chemiczny 93(8): 1472-1474.

Scheringer, M. (2008). "Nanoecotoxicology: Environmental risks of nanomaterials." Nature Nanotechnology 3(6): 322-323.

Schwarzenbach, R. P., P. M. Gschwend and D. M. Imboden (2005). Organic Liquid-Water Partitioning. Environmental Organic Chemistry, John Wiley \& Sons, Inc.: 213-244.

Sekine, R., G. Brunetti, E. Donner, K. Khaksar, K. Vasilev, A. K. Jämting, K. G. Scheckel, P. Kappen, H. Zhang and E. Lombi (2015). "Speciation and Lability of Ag-, AgCl-, and Ag2S-Nanoparticles in Soil Determined by X-ray Absorption Spectroscopy and Diffusive Gradients in Thin Films." Environmental Science \& Technology 49(2): 897-905.

Smoluchowski, M. (1917). "Versuch einer Mathematischen Theorie der Koagulations- Kinetik Kolloider Losungen." 92: 129. 
Sotiriou, G. A., C. Watson, K. M. Murdaugh, T. H. Darrah, G. Pyrgiotakis, A. Elder, J. D. Brain and P. Demokritou (2014). "Engineering safer-by-design silica-coated ZnO nanorods with reduced DNA damage potential." Environmental Science: Nano 1(2): 144-153.

Starnes, D. L., J. M. Unrine, C. P. Starnes, B. E. Collin, E. K. Oostveen, R. Ma, G. V. Lowry, P. M. Bertsch and O. V. Tsyusko (2015). "Impact of sulfidation on the bioavailability and toxicity of silver nanoparticles to Caenorhabditis elegans." Environmental Pollution 196: 239-246.

Stone, V., B. Nowack, A. Baun, N. van den Brink, F. von der Kammer, M. Dusinska, R. Handy, S. Hankin, M. Hassellov, E. Joner and T. F. Fernandes (2010). "Nanomaterials for environmental studies: Classification, reference material issues, and strategies for physico-chemical characterisation." Science of the Total Environment 408: 1745-1754.

Tandy, S., S. Mundus, J. Yngvesson, T. C. de Bang, E. Lombi, J. K. Schjørring and S. Husted (2011). "The use of DGT for prediction of plant available copper, zinc and phosphorus in agricultural soils." Plant and soil 346(1-2): 167-180.

Therezien, M., A. Thill and M. R. Wiesner (2014). "iogimportance of heterogeneous aggregation for NP fate in natural and engineered systems." Science of the Total Environment 485-486: 309-318.

Thomas, K., P. Aguar, H. Kawasaki, J. Morris, J. Nakanishi and N. Savage (2006). "Research Strategies for Safety Evaluation of Nanomaterials, Part VIII: International Efforts to Develop Risk-Based Safety Evaluations for Nanomaterials." Toxicological Sciences 92(1): 23-32. Tran, C. L., K. Donaldson, V. Stones, T. Fernandez, A. Ford, N. Christofi, J. G. Ayres, M. Steiner, J. F. Hurley, R. J. Aitken and A. Seaton (2005). A scoping study to identify hazard data needs for addressing the risks presented by nanparticles and nanotubes, Institute of Occupational Medicine: 1-48. Unrine, J. M., B. P. Colman, A. J. Bone, A. P. Gondikas and C. W. Matson (2012). "Biotic and Abiotic Interactions in Aquatic Microcosms Determine Fate and Toxicity of Ag Nanoparticles. Part 1. Aggregation and Dissolution." Environmental Science \& Technology 46: 6915-6924.

Unrine, J. M., W. A. Shoults-Wilson, O. Zhurbich, P. M. Bertsch and O. V. Tsyusko (2012). "Trophic Transfer of Au Nanoparticles from Soil along a Simulated Terrestrial Food Chain." Environmental Science \& Technology 46(17): 9753-9760.

Westerhoff, P. and B. Nowack (2012). "Searching for Global Descriptors of Engineered Nanomaterial Fate and Transport in the Environment." Accounts of Chemical Research 46(3): 844-853. Whitley, A. R., C. Levard, E. Oostveen, P. M. Bertsch, C. J. Matocha, F. von der Kammer and J. M. Unrine (2013). "Behavior of Ag nanoparticles in soil: Effects of particle surface coating, aging and sewage sludge amendment." Environmental Pollution 182: 141-149.

Zhang, W., Y. Yao, N. Sullivan and Y. Chen (2011). "Modeling the Primary Size Effects of Citrate-Coated Silver Nanoparticles on Their Ion Release Kinetics." Environmental Science \& Technology 45(10): 44224428. 